\title{
Whole-brain functional connectivity during acquisition of novel grammar: distinct functional networks depend on language learning abilities ${ }^{1}$
}

\author{
Olga Kepinska ${ }^{a, b,{ }^{*},}$ Mischa de Rover ${ }^{b, c, d}$, Johanneke Caspers ${ }^{a, b}$, \\ Niels O. Schiller, \\ a Leiden University Centre for Linguistics, Postbus 9515, 2300 RA Leiden, the Netherlands \\ ${ }^{\mathrm{b}}$ Leiden Institute for Brain and Cognition, c/o LUMC, Postzone C2-S, P.O.Box 9600, 2300 RC Leiden, the \\ Netherlands \\ ${ }^{c}$ Department of Anesthesiology, Leiden University Medical Center, Postzone P5-Q, P.O.Box 9600, 2300 RC Leiden, \\ The Netherlands \\ ${ }^{\mathrm{d}}$ Faculty of Social Sciences, Institute of Psychology, Clinical Psychology Unit, Pieter de la Court gebouw, \\ Wassenaarseweg 52 , \\ 2333 AK Leiden
}

* Corresponding author at Leiden University Centre for Linguistics, Postbus 9515, 2300 RA Leiden, the Netherlands, Tel. +31 (0)71 527 8530, e-mail: o.kepinska@hum.leidenuniv.nl

\footnotetext{
${ }^{1}$ A part of this study has been presented at the 22nd Annual Meeting of the Organization for Human Brain Mapping.
} 
ABSTRACT: In an effort to advance the understanding of brain function and organisation accompanying second language learning, we investigate the neural substrates of novel grammar learning in a group of healthy adults, consisting of participants with high and average language analytical abilities (LAA). By means of an Independent Components Analysis, a data-driven approach to functional connectivity of the brain, the fMRI data collected during a grammar-learning task were decomposed into maps representing separate cognitive processes. These included the default mode, task-positive, working memory, visual, cerebellar and emotional networks. We further tested for differences within the components, representing individual differences between the High and Average LAA learners. We found high analytical abilities to be coupled with stronger contributions to the task-positive network from areas adjacent to bilateral Broca's region, stronger connectivity within the working memory network and within the emotional network. Average LAA participants displayed stronger engagement within the task-positive network from areas adjacent to the righthemisphere homologue of Broca's region and typical to lower level processing (visual word recognition), and increased connectivity within the default mode network. The significance of each of the identified networks for the grammar learning process is presented next to a discussion on the established markers of inter-individual learners' differences. We conclude that in terms of functional connectivity, the engagement of brain's networks during grammar acquisition is coupled with one's language learning abilities.

KEYWORDS: language; learning; fMRI; functional connectivity; aptitude

\begin{abstract}
Abbreviations: AGL, artificial grammar learning; BOLD, blood-oxygenation-leveldependent; DMN, default mode network; EPI, echo-planar images; FEAT, FMRI Expert Analysis Tool; fMRI, functional magnetic resonance imaging; IC, independent component; ICA, independent component analysis; IFG, inferior frontal gyrus; L2, second language; LAA, language analytical ability; MELODIC, Multivariate Exploratory Linear Decomposition into Independent Components; PCA, principal component analysis; ROI, region of interest; SLA, second language acquisition; TE, echo time; TFCE, threshold-free cluster enhancement approach; TR, repetition time; VWFA, visual word form area
\end{abstract}

\title{
1. Introduction
}

The knowledge of a second language (L2) in today's globalised world seems more and more indispensable. High levels of proficiency in an L2 play an important role in many people's economic, social and private lives. No matter its importance however, language acquisition can be characterised by a great deal of variability in the rate, efficiency and ultimate success. Understanding the factors contributing to such variability can aid the efforts to describe the theoretical foundations of second language acquisition (SLA) and - from an applied point of view - in improving the outcomes of learning and instruction.

Like other high-level cognitive functions, language is governed by synchronized activity of distributed areas (cf. e.g. [1-6]). How different brain areas interact with each other, and what networks arise from those interactions is a question posed in more and more investigations into the neural architecture behind language processing and acquisition. Insights into the connectivity of the brain, both in terms of its structural (e.g. [7-13]) and functional networks (e.g. [14-23]), keep advancing our understanding of the neural foundations of human communication.

One powerful tool for exploring the connectivity of the brain is functional magnetic resonance imaging (fMRI). It offers a view on temporal correlations 
between the hemodynamic activity of different brain areas and can be used for visualising and quantifying functional connectivity patterns at rest or during cognitive tasks (cf. [22,24-29]).

With this paper, we wanted to explore the functional connectivity patterns during initial phases of L2 acquisition and networks' characteristics responsible for successful acquisition of a new language: a theme that has recently attracted interest in other studies in the context of language acquisition, which we briefly review below.

\subsection{Functional networks of the brain and individual differences in L2 acquisition}

Functional networks associated with vocabulary learning in a new language were investigated by Veroude et al. [22]. Functional connectivity of brain regions involved in phonological processing was measured during rest, before and after exposure to a new language. The authors showed that the recorded connectivity patterns differ between 'good' and 'bad' learners determined on the basis of their performance on a word recognition task after the scanning. Before the exposure to a new language, stronger connectivity between two sets of regions: the left supplementary motor area and the left precentral gyrus, and between the left insula and the left rolandic operculum was observed for 'good' compared to 'bad' learners. The authors interpreted this stronger connectivity as representing "a favourable disposition for the processing of the unknown language input" (p. 25). Furthermore, at the end of the task, good learners exhibited stronger functional connectivity between the left and right supramarginal gyrus, which was interpreted as an effect of exposure to the language itself and not a pre-existing difference between learners.

In a study investigating brain connectivity patterns in a group of Persian speakers learning new vocabulary in French, Ghazi Saidi et al. [18], showed that network integration levels decreased as proficiency for L2 increased, thus reflecting more automatic processing of the L2. Furthermore, Yang et al. [23] recently investigated the development of brain networks as a function of shortterm tonal L2 learning experience and reported functional connectivity results relating to successful acquisition of novel words. The successful learners in their study were identified on the basis of behavioural performance after the training, but due to their high accuracy on the sound discrimination task prior to training, they were hypothesized to be "well suited to learning a vocabulary in which lexical tones make up the critical information" (p. 45). At the level of brain connectivity, the successful learners demonstrated more integrated networks both before and after the training: in comparison to the non-learners, their frontal-temporal network was stronger at the first time-point, whereas at the second, they could be characterized by strong global, as well as local connectivity, and automatic lexical processing of acquired word knowledge driven by the inferior parietal lobule.

Learning novel words is undeniably one of the most important building blocks of acquisition of a new language. The brain's functional connectivity underlying another crucial subcomponent of language learning, namely the acquisition of grammatical rules, was investigated by Antonenko et al. [17] and Dodel et al. [21]. In their study, Antonenko et al. [17] used an artificial grammar learning (AGL) task to explore the ability to extract grammatical rules from new material in healthy older adults and found an opposite relationship between AGL task 
performance and resting-state functional connectivity of left and right BA 44/45: lower performance was tied to stronger inter-hemispheric functional coupling. Processing of syntax in L2 by bilinguals was explored by Dodel et al. [21] who showed that regions associated with syntax and language production - left inferior frontal gyrus (IFG), putamen, insula, precentral gyrus, and the supplementary motor area - were more functionally connected in L2 than in L1. The strength of this functional connectivity was modulated by participants' syntactic proficiency: the functional connectivity network was less present in less proficient bilinguals.

With the present experiment, we aimed at concentrating on novel grammar learning, and capturing whole-brain functional connectivity correlates of the process of new syntax acquisition in its initial phase. Similarly to Antonenko et al. [17], we employed an AGL paradigm in order to ensure that our data represent the neurobiology of syntax acquisition and processing, without the interference of semantics, phonology or pragmatics, and are not influenced by prior exposure (cf. e.g. [30-32]). In particular, we chose a paradigm enabling an investigation into rule learning in real time, in which learning is simultaneous to the recording of fMRI data. The chosen artificial language BROCANTO [33-39] is based on a set of pronounceable pseudo words, combined in ways following rules found in many natural languages. The paradigm can thus be seen as a model for language learning, though a highly controlled one (see section 2.2 for a further description of the paradigm).

\subsection{Language aptitude and language analytical ability}

Next to investigating the functional connectivity patterns present during the acquisition of novel grammar, we wanted to explore more fully, what in previous language acquisition connectivity studies was interpreted as a "favourable disposition" [22] of L2 learners or being "well suited" to learn a particular aspect of an L2 [23]. Within the field of second language acquisition, a bulk of research has been dedicated to investigating pre-existing differences between learners, and L2 learning success has repeatedly been linked to the notion of language aptitude (cf. e.g. [40-42]).

Language aptitude is defined as an individual, relatively immutable cognitive ability particular to language learning, which is a combination of skills that are fairly independent from each other. The multi-componential nature of language aptitude can be found in tests measuring it. For example, the LLAMA Language Aptitude Test (LLAMA) [43], consists of four parts: (1) a vocabulary learning task, (2) a test of phonetic memory, (3) a test of sound-symbol correspondence and (4) a test of grammatical inferencing, being a measure of language analytical ability (LAA). LAA is relevant for pattern identification during SLA, which involves analysing and processing new linguistic input [44]. Arguably, LAA is the most important of the language aptitude components for grammar learning: learners with high degrees of LAA are sensitive to the grammatical structure of new languages and are able to make linguistic generalisations easily. According to SLA research, LAA plays an important role in L2 acquisition in a variety of settings, such as immersion [45], classroom [46] and lab [47].

With our study, instead of defining successful learners post-hoc, on the basis of their behavioural performance in the task at hand, we wanted to concentrate on neural substrates of the pre-existing differences between learners, diagnosed prior to the task, by means of a standardised test instrument, and in this way explore the neural underpinnings of a "favourable disposition" (cf. [22]) for novel 
grammar learning. We manipulated the language analytical abilities of our participants by recruiting them on the basis of the LLAMA sub-test measuring LAA. Two groups participated in the experiment: with high and average LAA.

\subsection{Whole-brain functional connectivity approach}

Functional connectivity of the brain - temporal correlations between the hemodynamic activity of different areas - can be investigated in various ways by means of fMRI. For example, the studies cited in section 1.1 above, resorted to an approach employing a set of pre-defined regions of interest (ROIs). Though such a method is undoubtedly informative as to the insights concerning the cooperation between the given brain areas, it also limits the observations to the regions examined. The goal of the current study was therefore to approach the connectivity question in a data-driven manner and look for the intrinsic organisation of the brain as different networks without a priori spatial constraints.

A method allowing for such an approach is the independent component analysis (ICA) of fMRI data. This data-driven technique allows for detection of structured spatiotemporal processes in neuroimaging data [48] by decomposing the data into a set of spatially independent activation maps (components) and their time courses. The components can reflect meaningful neuronal signal sources (i.e., networks), as well as noise or artefacts. The meaningful components are seen as a representation of interconnected networks of brain regions that co-activate when certain types of tasks or cognitive processes are being performed. Several well-established and highly reproducible [25] functional networks are typically reported in the functional neuroimaging literature, both in resting-state (see e.g. $[24,25,28,49])$ and task-related investigations (see e.g. [28,50]). They represent e.g. the default mode network (DMN) of the brain, motor processing, visual processing, cognition, perception, attention, and emotional processing (cf. $[24,28,51,52])$.

After extraction, components of interest are subject to identification and interpretation by the researcher and inter-individual differences within each component can be assessed statistically. For a detailed description of the applied ICA methodology, see section 2.4.3 below and e.g. Beckmann and Smith [24], Beckmann et al. [48,53], and Veer [54].

\subsection{Hypotheses}

The aim of this study was to examine the characteristics of networks present during the grammar learning process and their features representing preexisting differences between learners. It remains to be noted that previous studies investigating language learning concentrated predominantly on predefined regions of interest and their (mutual) connectivity, thus not fully profiting from the wealth of information present in such data. We approached the question in a data-driven way, adopting an exploratory approach by investigating whole-brain functional connectivity during the process of learning.

Based on current functional connectivity literature, we expect that the spatiotemporal characteristics of grammar learning on the neural level can be distinguished by several substrates, both typical to the type of the received input, as well as representing the highly reproducible intrinsic organisation of the brain found in resting-state and task-related investigations. 
In terms of the differences between groups of learners chosen on the basis on their language analytical abilities, on the whole stronger functional connectivity patterns are expected in case of the highly skilled learners. In particular, we expect the syntax-related regions such as left IFG, putamen, insula, precentral gyrus, and the supplementary motor area to contribute more to the networks driven by the type of input in case of the highly skilled learners. Following from Antonenko et al.'s [17] study, we further expect bilateral contribution from the Broca's area and its contra-lateral homologue in case of the moderately skilled learners.

\section{Materials and methods}

Data for this experiment were collected together with data reported in [55]. The present study concentrated on functional connectivity fMRI data, not described elsewhere. Participants' demographic details and task details remain the same for both studies. For reasons of clarity they are shortly presented hereunder.

\subsection{Participants}

Two groups of participants (with high and average LAA) were determined by administering a language aptitude test, the Llama Language Aptitude Test (LLAMA) [43], to a large group of participants $(N=307)$. On the basis of the test, forty-two healthy adults with no contra-indications for an MRI scan were chosen to take part in the MRI experiment.

There were 20 participants in the Average LAA group (16 female) ${ }^{2}$, age 19-39 years $(M=23.60$ years) and 22 participants in the High LAA group (16 female), age 19-43 years $(M=23.18$ years). All participants were native speakers of Dutch, right-handed and had normal or corrected-to-normal vision.

The Medical Ethical Committee of the Leiden University Medical Centre (LUMC) (Leiden, the Netherlands) approved the protocol of the MRI experiment; behavioural testing was also conducted according to the Ethics Code of the Faculty of Humanities at Leiden University. Participants gave written informed consent prior to the experiment and were remunerated for their time.

\subsection{Stimuli and design}

The stimulus material was created on the basis of the artificial grammar of BROCANTO [33-39], the schematic representation of which can be found in Figure 1. The AGL task was administered in the scanner and consisted of three blocks of learn and test phases. Six days after the fMRI experiment, participants performed a delayed transfer test. The stimulus material consisted of both grammatical and ungrammatical sentences. The grammatical ones were used in the learning phases of the experiment, the test phases and the transfer test contained both grammatical and ungrammatical sentences. The learning phases, during which the neuroimaging data reported in this paper were collected,

\footnotetext{
2 Although a comparison of highly skilled learners against a group of low aptitude learners would probably yield more clear-cut results, the low aptitude LAA learners were very infrequent in our sample. Only 8 Dutch-speaking participants scored within low range (0-15, according to the manual of the LLAMA test [43]) on the LLAMA_F test. Hence, the group of learners with high LAA was compared to a group who scored within average range.
} 
consisted of forty grammatically correct sentences. The sentences were presented in a random order, for 8 seconds each, preceded by a fixation cross (3 seconds). Participants were instructed to discover the grammatical rules of the language by analysing the correct sentences in the learning phases, and to give a grammaticality judgment in the test phases. Visual feedback was provided for the grammaticality judgements.

For a further description of the version of BROCANTO used in this experiment and technical presentation details see [55].

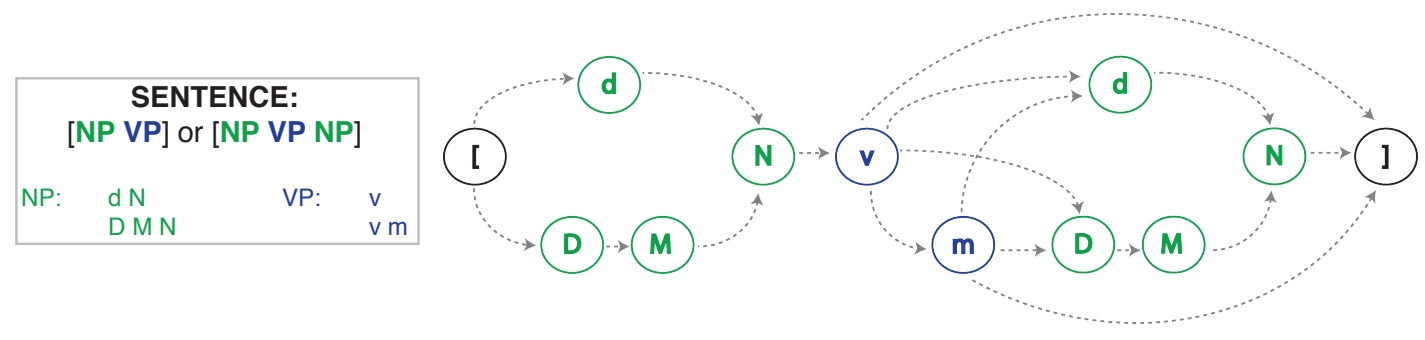

POSSIBLE SENTENCES:

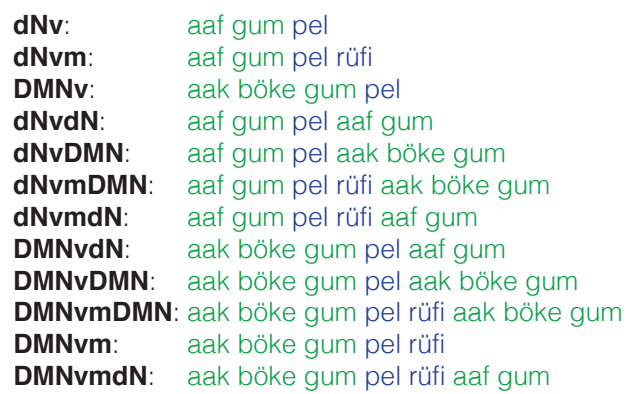

VIOLATIONS:

determiner-noun-agreement

(DN instead of $\mathrm{dN}$, and $\mathrm{dMN}$ instead of $\mathrm{DMN}$ ):

*aak gum pel aaf gum instead of aaf gum pel aaf gum

word class repetitions of nouns or verbs

( $\mathrm{NN}$ instead of $\mathrm{Nv}$, and $\mathrm{vv}$ instead of $\mathrm{Nv}$ )

*aaf prez pel aaf gum instead of aaf gum pel aaf gum

phrase structure violations

(NP NP instead of NP VP, and NP NP VP instead of NP VP NP)

*aaf gum aaf gum pel instead of aaf gum pel aaf gum

Figure 1. Representation of the artificial grammar of BROCANTO and its possible sentences. The graph in the top panel summarises the grammatical rules according to which the sentences were created: the nodes specify word classes ( $\mathrm{N}=$ noun ("gum", "trul"), v = verb ("pel", "prez"), $\mathrm{M}=$ adjective ("böke"), $\mathrm{m}=$ adverb ("ruff"), $\mathrm{d}$ ("aaf") and $\mathrm{D}$ ("aak") = determiner), and the arrows represent possible transitions between nodes. The green elements form noun phrases, the blue ones verb phrases. A grammatical sentence is formed by a transition from beginning ([) to end (]), cf. [33-39]. The bottom panel lists all types of the sentences built according to the rules together with examples. On the left, three types of violations are exemplified.

\subsection{Data acquisition}

Imaging data were acquired using a Philips 3T MR-system (Best, The Netherlands) located at the Leiden University Medical Centre (LUMC) equipped with a SENSE-32 channel head coil. Three fMRI sequences were acquired for every participant, each during the consecutive learning phase of the AGL task. We obtained echo-planar images (EPI) using a T2*-weighted gradient echo sequence (repetition time $[\mathrm{TR}]=2200 \mathrm{~ms}$, echo time $[\mathrm{TE}]=30 \mathrm{~ms}$, matrix size: 80 x 80, 38 axial slices, $2.75 \times 2.75 \times 2.75 \mathrm{~mm}$ (+ $10 \%$ slice gap) voxel size). EPIs were scanned parallel to the anterior-posterior commissure plane. The length of each scan sequence was 200 volumes and lasted 7.5 minutes. Anatomical imaging included a 3D gradient-echo $\mathrm{T} 1$-weighted sequence $(\mathrm{TR}=9.755 \mathrm{~ms}, \mathrm{TE}=$ $4.59 \mathrm{~ms}$; matrix 256 × 256; voxel size: $1.2 \times 1.2 \times 1.2 \mathrm{~mm}$; 140 slices) and a high- 
resolution $\mathrm{T} 2$-weighted image $(\mathrm{TR}=2200 \mathrm{~ms}$, $\mathrm{TE}=30 \mathrm{~ms}$; matrix $112 \times 112$; voxel size: $2.0 \times 2.0 \times 2.0 \mathrm{~mm}$; 84 slices).

\subsection{Data analysis}

\subsubsection{Behavioural data}

Based on the individual hit- and false-alarm rates, the responses on the AGL task for each participant were transformed into d' scores. Within the framework of Signal Detection Theory [56] d' scores are used to represent a measure of sensitivity that accounts for response bias. The d'scores were calculated by subtracting the normalised false-alarm rates from normalised hit rates (see Macmillan \& Creelman [56] for details).

Following previous studies employing similar experimental designs [33-39], the d' scores were then analysed with a repeated measures ANOVA (alpha level = 0.05) with group as a between-subject factor (High LAA vs. Average LAA) and learning phase (first phase, second phase, last phase and transfer test) as a within-subject factor. As imaging data from two subject had to be discarded due to technical reasons (see section 2.4.2 below), the behavioural data report on the same sample of 40 participants. The analysis was performed in SPSS version 22 [57]. Mauchley's test showed violations of sphericity against the factor phase, $\mathrm{X}^{2}(5)=19.957, \quad p<.01$, therefore Greenhouse-Geisser correction for nonsphericity was used $(\varepsilon=.772)$.

\subsubsection{Pre-processing of imaging data}

Imaging data from one subject from the High LAA group were discarded from further analyses due to an artefact in one of the scans; another was rejected due to excessive motion.

The remaining forty data sets (from twenty participants in each group) acquired during the learning phases of the AGL task were processed using FSL software Version 5.0.7 (FMRIB's Software Library, www.fmrib.ox.ac.uk/fsl [58]). FEAT (FMRI Expert Analysis Tool) Version 6.00 (www.fmrib.ox.ac.uk/fsl), implemented in MELODIC (Multivariate Exploratory Linear Decomposition into Independent Components) Version 3.14, was used for pre-processing. The following prestatistics processing was applied: motion correction using MCFLIRT [59], nonbrain removal using BET [60], spatial smoothing using a Gaussian kernel of FWHM $5 \mathrm{~mm}$, grand-mean intensity normalisation of the entire 4D dataset by a single multiplicative factor, high-pass temporal filtering (Gaussian-weighted least-squares straight line fitting, with sigma $=50.0 \mathrm{~s}$ ).

The functional images were registered to MNI-152 standard space (T1-standard brain averaged over 152 subjects; Montreal Neurological Institute, Montreal, QC, Canada) using a three-step registration from functional to high-resolution structural T2-image (rigid body, 6 degrees of freedom) to T1-image (rigid body, 6 degrees of freedom) to MNI-template (affine registration, 12 degrees of freedom). Registration was carried out using FLIRT [59,61].

\subsubsection{Extraction of functional connectivity networks}

Group ICA was carried out using multi-session Tensor-ICA [24] as implemented in MELODIC Version 3.14. Tensor-ICA allowed for a model-free fMRI analysis of the three AGL phases by means of a three-way data decomposition into independent components (ICs), representing signal or artefacts in the data in 
terms of time courses, spatial maps and session/subject modes (see Beckmann \& Smith [53] for a detailed description of Tensor-ICA).

The following default ICA processing steps were applied: masking of non-brain voxels, voxel-wise de-meaning of the data and normalisation of the voxel-wise variance. Subsequently, the data set was projected into a 10-dimensional subspace using principal component analysis (PCA): in order to approximate earlier studies [24,28], the dataset was decomposed into 10 sets of vectors, which describe signal variation across the temporal domain (time-courses), the session/subject domain and across the spatial domain (maps) by optimising for non-Gaussian spatial source distributions using a fixed-point iteration technique [62]. Estimated component maps were divided by the standard deviation of the residual noise and thresholded by fitting a mixture model to the histogram of intensity values [63]. Finally, a grey matter mask was used to mask out non-grey matter regions in each of the 10 obtained IC maps. FSL Cluster tool was subsequently used to define significant clusters comprising the ICs, with the threshold of $Z>2.3$.

Prior to the higher-level statistical analysis the ten IC maps were inspected visually in order to distinguish anatomically and functionally task-relevant networks. Four out the ten components were identified as artefacts resulting from head motion, fluctuations in cerebrospinal fluid, and physiological or scanner noise. Visual maps of the noise components are presented in the supplementary data. The first three visual maps all show activity at the edges of the brain, which is typical for motion and scanner drift artefacts. The fourth one, in addition to showing activity on the edges of the brain, depicts activation that is dispersed and does not form a coherent network from an anatomical point of view. One task-relevant IC map represented a visual processing network (see component (d) in Table 2 and Figure 2), not relevant for our research questions. In order to maximise the statistical power of the group comparisons, only five components of interest were included in the between-group analysis ${ }^{3}$.

\subsubsection{Statistical analysis}

Subject-specific versions of the spatial maps, and associated timeseries were created on the basis of the spatial maps from the group-average analysis in order to test for differences between the High and Average LAA groups in the identified components. We adopted a dual regression procedure [64,65] which uses the network time-course in a temporal and spatial regression against individual fMRI data. First, each component was spatially masked with a binary representation of the group main effects image. Then, for each subject, the groupaverage set of spatial maps was regressed (as spatial regressors in a multiple regression) into the subject's $4 \mathrm{D}$ space-time dataset. This resulted in a set of subject-specific timeseries, one per group-level spatial map. Subsequently, those timeseries were regressed (as temporal regressors, again in a multiple regression) into the same $4 \mathrm{D}$ dataset, resulting in a set of subject-specific spatial maps, one per group-level spatial map.

\footnotetext{
3 An exploratory independent sample t-test (implemented in FSL's Randomise tool, Version 2.9) performed with permutation testing with 5,000 permutations, revealed, however, that the two groups did not differ in the degree of engagement of the visual processing network $(p>.05$, Bonferroni corrected, for both High LAA > Average LAA and Average LAA > High LAA contrasts).
} 
The resulting spatial maps represented a measure of the degree to which bloodoxygenation-level-dependent (BOLD) signal fluctuations in each voxel co-varied with each IC time series for each subject separately. In other words, each participant's spatial map for a given IC was seen as a voxelwise map of the strength of functional connectivity with that IC. The spatial maps were then used in voxelwise analysis to assess differences between the Average and the High LAA groups in connectivity strength within the identified ICs. FSL's Randomise tool, Version 2.9, was used to perform non-parametric permutation testing with 5,000 permutations. The resulting contrast maps for each taskrelevant independent component were thresholded with a threshold-free cluster enhancement approach (TFCE) [66], a standard and conservative approach for correcting for multiple comparisons with regard to the number of voxels in the brain (at $p<.05$ ). Subsequently we corrected for the number of investigated components (using a Bonferroni correction).

\section{Results}

\subsection{Behavioural data}

The d' scores on the test phases of the AGL task in both High LAA and Average LAA groups increased over the course of the experiment, see Table 1. A repeatedmeasures ANOVA showed a main effect of learning phase, $F(2.316,88.018)=38.224, p<.001, \eta_{\mathrm{p}}{ }^{2}=.501$, and a significant effect of group, $F(1,38)=14.318, p=.001, \eta_{\mathrm{p}}^{2}=.274$. The High LAA group performed better than the Average LAA group, and there was a significant interaction between group and phase, $F(2.316,88.018)=4.896, p<.01, \mathrm{np}^{2}=.114$.

\begin{tabular}{c|c|c}
\multirow{2}{*}{ AGL phase: } & \multicolumn{2}{|c}{ Mean (SD) } \\
\cline { 2 - 3 } & High LAA & Average LAA \\
\hline $\mathbf{1}$ & $1.20(0.83)$ & $0.79(0.89)$ \\
\hline $\mathbf{2}$ & $2.52(1.02)$ & $1.43(1.28)$ \\
\hline 3 & $2.88(0.86)$ & $1.90(1.10)$ \\
\hline transfer & $2.92(0.86)$ & $1.45(1.0)$
\end{tabular}

Table 1. Proportion correct responses (and Standard Deviations) for the three phases of the AGL task and the subsequent transfer test for High and Average LAA groups.

\subsection{Functional connectivity networks involved in learning language rules}

Six functionally relevant functional connectivity networks were found using the group Independent Components Analysis. A summary of the cluster peaks in each of the networks can be found in Table 2; Figure 2 presents the results of the analysis overlaid on the MNI-152 standard brain. The final thresholded maps can be classified as follows:

(a) Task-positive/language network: containing seven clusters (peaks described in Table 2). These clusters were extending into the set of areas consistent with the task-positive network (cf. [49]), with the addition of task-specific, i.e. language network activations. Moreover, the regions constituting this IC largely overlap with the set of areas described by Dodel et al. [21] as syntaxrelated. The areas found in the task-positive network are the superior and middle prefrontal cortices, paracingulate gyrus and ventrolateral prefrontal cortex [24]. Also involved in the task-positive network are the activated 
parietal structures (supramarginal and angular gyrus). The bilateral activation of caudate nuclei can be attributed to their role in learning and memory (cf. [67,68]), and to their role as a centre for language control (cf. [69-71]). Furthermore, there was activity in the insula, which, having direct connections to Broca's area and other speech and language centres, is implicated in coordinating higher-order cognitive aspects of speech and language production [72]. Note that the activations in this network were mostly bilateral (see Figure 2), with asymmetry to the left hemisphere.

(b) Default-mode network: activity was found in the anterior and posterior cingulate gyrus, in the frontal pole, frontal orbital cortex, superior parietal and temporal region and in the hippocampus - areas described as belonging to the DMN [24,25,73-76]. In addition, there was activation in the cerebellum, which in a number of resting-state functional connectivity studies was shown to participate in higher-order networks such as the DMN [77-81]. Also observed was the contribution of the subcallosal cortex.

(c) Working memory network: activations were consistent with the working memory network as described by Owen, McMillan, Laird, \& Bullmore [82]. These included: bilateral frontal pole and prefrontal cortex, cingulate gyrus, premotor cortex and posterior parietal cortex, including precuneus. Moreover, there was activity in the superior parietal lobule, which has been shown to be critically important for the manipulation of information in working memory [83]; the supramarginal gyrus (here activated bilaterally), has been reported to be involved in verbal working memory [84]. Similarly, the bilateral activation of the thalamus can be related to its enhanced activation in verbal working memory tasks [82]. Additionally, the network comprised of the left temporal occipital fusiform cortex, the activation of which corresponded to the localization visual word form area (VWFA), shown to be involved in the recognition of words and letters from simple shape images [85,86].

(d) Visual areas: including activations in the lateral occipital cortex and occipital pole.

(e) Cerebellar network: cerebellum was found to interact with the right IFG and the basal ganglia structures, pallidum and putamen. This assembly of structures has been described as part of the procedural learning system by Ullman [87] (see also [88,89]). Also observed was activation of the thalamus, the right temporal pole and the left temporal fusiform gyrus.

(f) Emotional network: activity was found in the subcortical structures of brainstem (including the inferior olivary nucleus and the red nucleus), amygdala and the left hippocampus; cortically, the right temporal pole, bilateral parietal operculum, anterior cingulate gyrus, right precentral gyrus, the left posterior middle temporal gyrus and the subcallosal cortex were activated. This pattern of functional connectivity largely overlaps with the regions together forming "the emotional brain" circuitry, dedicated to processing and regulating emotions [90,91]. 


\section{Cortical region (peak)}

L/R

(a) Task-positive/Language Network

(1) Lateral Occipital Cortex, superior division / Superior Parietal Lobule

(2) Precentral Gyrus / Middle Frontal Gyrus

(3) Middle Frontal Gyrus / Frontal Pole

(4) Paracingulate Gyrus

(5) Insular Cortex / Frontal Orbital Cortex

(6) Caudate

(7) Caudate

\begin{tabular}{cccccc}
$\mathrm{L}$ & 18874 & -30 & -62 & 46 & 7.67 \\
$\mathrm{~L}$ & 6758 & -40 & 4 & 32 & 7.67 \\
$\mathrm{R}$ & 5076 & 50 & 32 & 26 & 7.27 \\
$\mathrm{~L}$ & 1237 & -6 & 16 & 46 & 6.12 \\
$\mathrm{R}$ & 205 & 32 & 24 & -2 & 4.84 \\
$\mathrm{~L}$ & 151 & -16 & 6 & 8 & 2.91 \\
$\mathrm{R}$ & 49 & 16 & 6 & 14 & 2.77 \\
\hline
\end{tabular}

(b) Default-mode Network

\begin{aligned} & \hline (1) Frontal Pole \\ & \hline (2) Cingulate Gyrus, posterior division / Precuneus \\ & Cortex \\ & \hline (3) Frontal Orbital Cortex \\ & \hline (4) $\begin{array}{l}\text { Angular Gyrus / Lateral Occipital Cortex, } \\ \text { superior division }\end{array} \\ &$\hline (5) Lateral Occipital Cortex, superior division \\ & \hline (6) $\begin{array}{l}\text { Middle Temporal Gyrus / Superior Temporal } \\ \text { Gyrus, posterior division }\end{array} \\ &$\hline (7) Frontal Orbital Cortex \\ & \hline (8) Subcallosal Cortex \\ & \hline (9) Cerebellum \\ & \hline (10) Cerebellum \\ & \hline (11) Hippocampus \\ & \hline (12) Hippocampus \\ & \hline (13) Occipital Pole / Cuneal Cortex \\ & \hline (14) Parahippocampal Gyrus / Temporal Fusiform \\ & Cortex, posterior division / Lingual Gyrus / \\ & Temporal Occipital Fusiform Cortex \\ & \hline\end{aligned}

\begin{tabular}{lccccc}
\hline- & 10339 & 0 & 58 & 0 & 6.48 \\
$\mathrm{~L}$ & 5065 & -6 & -54 & 28 & 6.29 \\
$\mathrm{R}$ & 1981 & 44 & 26 & -14 & 4.09 \\
$\mathrm{R}$ & 1828 & 56 & -58 & 28 & 4.88 \\
$\mathrm{~L}$ & 1658 & -48 & -72 & 32 & 5.46 \\
$\mathrm{~L}$ & 1300 & -62 & -16 & -12 & 4.59 \\
$\mathrm{~L}$ & 255 & -40 & 24 & -18 & 3.82 \\
$\mathrm{R}$ & 209 & 2 & 12 & -6 & 3.98 \\
$\mathrm{~L}$ & 161 & -28 & -82 & -34 & 3.77 \\
$\mathrm{R}$ & 140 & 32 & -82 & -34 & 3.62 \\
\hline $\mathrm{R}$ & 57 & 28 & -22 & -18 & 3.4 \\
\hline $\mathrm{L}$ & 43 & -24 & -22 & -20 & 3.02 \\
\hline $\mathrm{R}$ & 37 & 8 & -88 & 28 & 2.79 \\
\hline & 33 & -26 & -40 & -14 & 2.88
\end{tabular}
Temporal Occipital Fusiform Cortex

(c) Working Memory Network

(1) Supramarginal Gyrus, anterior division / Parietal Operculum Cortex / Planum Temporale

(2) Supramarginal Gyrus, anterior division / Parietal Operculum Cortex / Postcentral Gyrus

$\mathrm{L}$

Precuneus Cortex/Intracalcarine Cortex / Supracalcarine Cortex

(4) Frontal Pole

(5) Frontal Pole / Middle Frontal Gyrus

(6) Lateral Occipital Cortex L L

(7) Thalamus

(8) Thalamus

(9) Superior Parietal Lobule / Postcentral Gyrus

(10) Superior Frontal Gyrus triangularis

\begin{tabular}{llllllll}
\hline (12) Temporal Occipital Fusiform Cortex & $\mathrm{L}$ & 30 & -28 & -58 & -18 & 2.52 \\
\hline
\end{tabular}

\begin{tabular}{llllllll}
\hline (13) & Postcentral Gyrus / Superior Parietal Lobule & R & 27 & 34 & -36 & 56 & 2.45 \\
\hline
\end{tabular}

(d) Visual Network

(1) Occipital Pole / Lateral Occipital Cortex, superior division

(2) Lateral Occipital Cortex, superior division / Superior Parietal Lobule

$\begin{array}{lccccc}\mathrm{L} & 17372 & -18 & -90 & 18 & 3.7 \\ \mathrm{~L} & 15 & -16 & -60 & 54 & 2.39\end{array}$

(e) Cerebellar Network

(1) Cerebellum

(2) Temporal Pole

(3) Pallidum / Putamen

(4) Temporal Fusiform Cortex, anterior division /

Temporal Fusiform Cortex, posterior division

(5) Inferior Frontal Gyrus, pars opercularis

(6) Thalamus

\begin{tabular}{lccccc}
$\mathrm{L}$ & 28906 & -4 & -64 & -22 & 6.36 \\
$\mathrm{R}$ & 108 & 52 & 10 & -20 & 3.07 \\
\hline $\mathrm{L}$ & 81 & -26 & -16 & -2 & 3.11 \\
$\mathrm{~L}$ & 36 & -34 & -6 & -38 & 2.79 \\
$\mathrm{R}$ & 13 & 62 & 14 & 4 & 2.66 \\
\hline $\mathrm{L}$ & 10 & -4 & -2 & -4 & 2.5 \\
\hline
\end{tabular}




\begin{tabular}{|c|c|c|c|c|c|c|}
\hline \multirow{2}{*}{ Cortical region (peak) } & \multirow{2}{*}{ L/R } & \multirow{2}{*}{$\begin{array}{c}\text { Size } \\
\text { (voxels) }\end{array}$} & \multicolumn{3}{|c|}{ Peak location } & \multirow{2}{*}{$Z_{\max }$} \\
\hline & & & 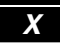 & $Y$ & $\mathbf{Z}$ & \\
\hline \multicolumn{7}{|l|}{ (f) Emotional Network } \\
\hline (1) Brain-Stem & $\mathrm{L}$ & 14571 & -14 & -28 & -28 & 9.85 \\
\hline (2) Temporal Pole & $\mathrm{R}$ & 881 & 44 & 10 & -16 & 6.1 \\
\hline (3) Parietal Operculum Cortex & $\mathrm{R}$ & 90 & 42 & -30 & 20 & 3.18 \\
\hline (4) Postcentral Gyrus / Precentral Gyrus & $\mathrm{R}$ & 49 & 52 & -10 & 32 & 2.7 \\
\hline (5) Cingulate Gyrus, anterior division & - & 23 & 0 & -8 & 30 & 2.65 \\
\hline (6) Middle Temporal Gyrus, posterior division & $\mathrm{L}$ & 17 & -48 & -28 & -10 & 2.75 \\
\hline (7) Parietal Operculum Cortex / Planum Temporale & $\mathrm{L}$ & 12 & -52 & -34 & 18 & 2.65 \\
\hline
\end{tabular}

Table 2. Six functionally relevant Independent Components distinguished by the group TensorICA analysis. Each IC represents an assembly of regions associated with distinct cognitive processes at play during AGL. Only clusters of 10 or more voxels are reported; $\mathrm{x}, \mathrm{y}$ and $\mathrm{z}$ coordinates are in MNI space, regions labelled according to Harvard-Oxford Cortical and Subcortical Structural Atlases and the Juelich Histological Atlas (all implemented within FSLVIEW, part of FSL). 
(a)

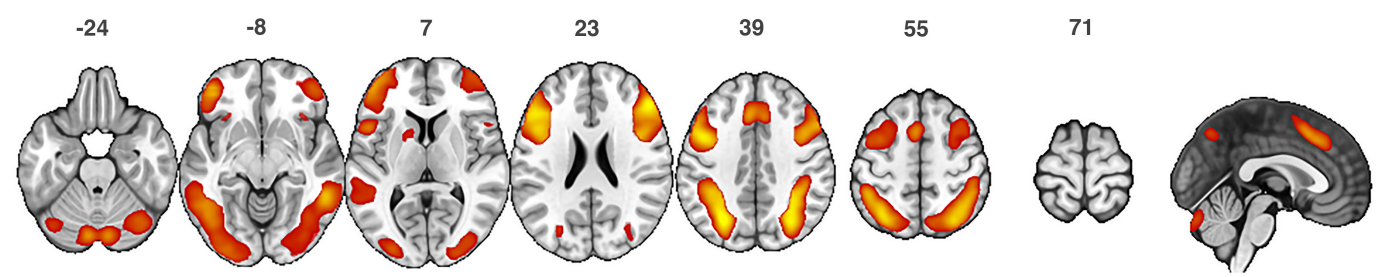

(b)

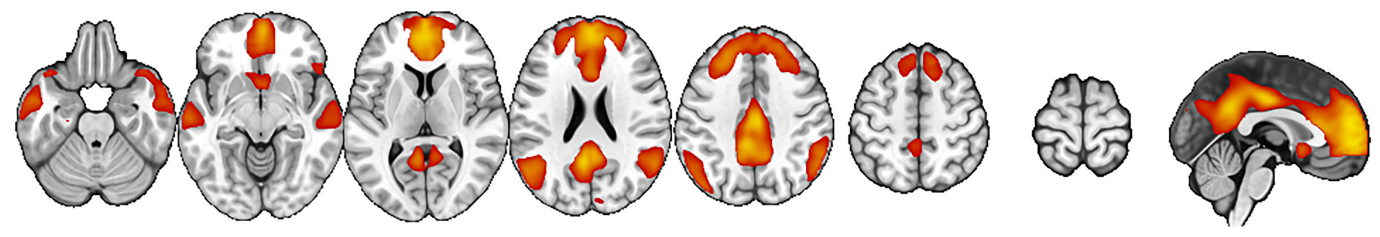

(c)

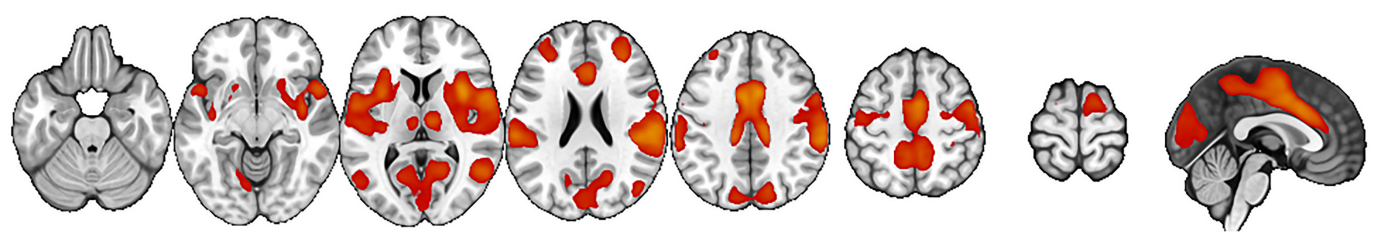

(d)

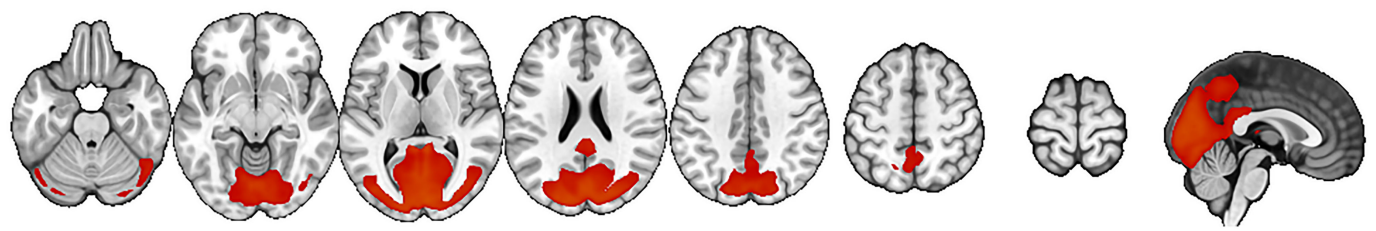

(e)

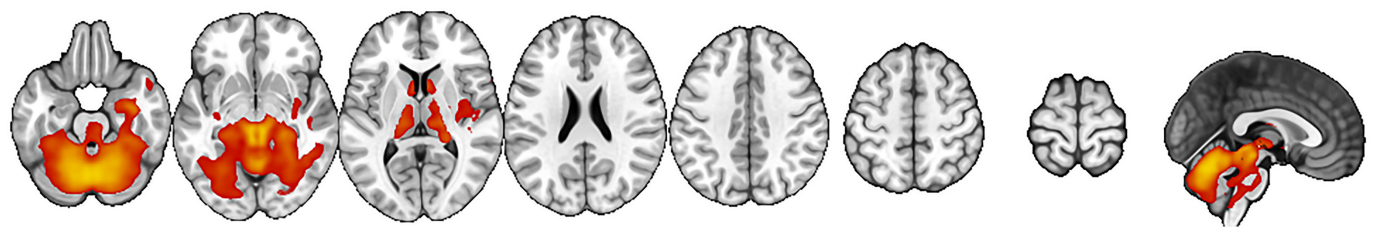

(f)

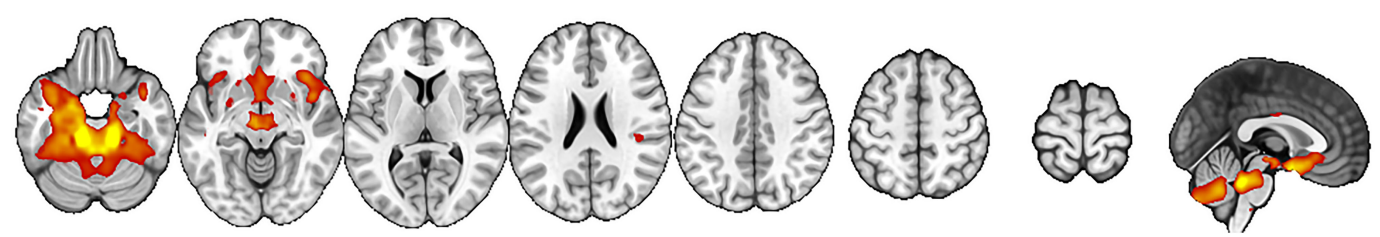

Figure 2. Group ICA networks. Depicted here are the six functional connectivity networks resulting from the group ICA step carried out on the concatenated data sets from both High and Average LAA groups. Images are z-statistics, ranging from 2.3 (red) to 7 (yellow), overlaid on the MNI-152 standard brain. The left hemisphere of the brain corresponds to the left side in the image. Brain activations are displayed using MRIcroGL (http://www.mccauslandcenter.sc.edu/mricrogl/). 


\subsection{Group differences}

Five IC networks were subject to a between-group analysis: since no differences between the groups were expected in visual processing, the component (d) (see Table 2 and Figure 2) was excluded from the analysis in order to maximise the statistical power of the group comparisons. All investigated networks were significantly represented in both Average and High LAA groups (mean connectivity maps per group, showing a significant representation of each IC in the two groups are presented in the supplementary materials). Between-group differences in the voxel-wise spatial distribution of the functional connectivity maps were revealed in four out of five ICs, which were subject to two-sample unpaired t-tests, corrected for multiple comparisons (at $p<.05$, TFCE-corrected) and for the number of investigated components, thus resulting in $p<.01$ (see Table 3).

In the first IC (the task-positive or language network), the Average LAA group displayed stronger functional connectivity in comparison with the High LAA in two clusters: (1) the right precentral gyrus bordering on pars opercularis in the IFG and (2) right occipital fusiform gyrus. The High LAA group displayed stronger connectivity within this network bilaterally, in two clusters adjacent to Broca's area and its right-hemisphere homologue: (1) left middle frontal gyrus, precentral gyrus and IFG, pars opercularis and (2) right IFG, pars opercularis and middle frontal gyrus (see Figure 3a).

Three clusters of activity were found to have a stronger contribution to the default-mode network for the Average as compared to the High LAA group. They were localised in (1) the posterior cingulate gyrus, (2) paracingulate gyrus and anterior cingulate gyrus and in the (3) right frontal pole and middle frontal gyrus (Figure 3b).

Central opercular cortex in the right hemisphere was found to have and increased functional connectivity for the High as compared to the Average LAA group in the third component (working memory network) (Figure 3c). Similarly, the High LAA group displayed increased connectivity in the emotional network component in two clusters: (1) the right amygdala and (2) mammillary body in the right hemisphere (Figure 3d). 


\section{Cortical region (peak)}

(a) Task-positive/Language Network

Average LAA > High LAA

(1) Precentral Gyrus / Inferior Frontal Gyrus, pars opercularis

(2) Occipital Fusiform Gyrus / Lateral Occipital Cortex inferior division

$\begin{array}{cccccc}\mathrm{R} & 57 & 50 & 4 & 22 & p<0.001 \\ \mathrm{R} & 24 & 36 & -78 & -18 & p<0.01\end{array}$

High LAA > Average LAA

(1) Middle Frontal Gyrus / Precentral Gyrus / Inferior Frontal Gyrus, pars opercularis

(2) Inferior Frontal Gyrus, pars opercularis / Middle Frontal Gyrus

\begin{tabular}{llllll}
$\mathrm{L}$ & 11 & -42 & 8 & 34 & $p<0.01$ \\
$\mathrm{R}$ & 3 & 42 & 16 & 26 & $p<0.01$ \\
\hline
\end{tabular}

\begin{tabular}{lcccccc}
\hline (b) Default-mode Network & & & & & & \\
\hline Average LAA > High LAA & L & 29 & -2 & -36 & 32 & $p<0.01$ \\
\hline (1) Cingulate Gyrus, posterior division & $\mathrm{R}$ & 11 & 6 & 34 & 28 & $p<0.01$ \\
\hline $\begin{array}{l}\text { (2) Paracingulate Gyrus / Cingulate Gyrus, anterior } \\
\text { division }\end{array}$ & $\mathrm{R}$ & 4 & 30 & 40 & 42 & $p<0.01$ \\
\hline (3) Frontal Pole / Middle Frontal Gyrus & & & & & & \\
\hline
\end{tabular}

\begin{tabular}{lllllll}
\hline High LAA > Average LAA & & & & & & \\
\hline- & - & - & - & - & - & - \\
\hline
\end{tabular}

\begin{tabular}{lllllll}
\hline (c) Working Memory Network & & & & & & \\
\hline Average LAA > High LAA & - & - & - & - & - & - \\
\hline- & & & & & & \\
\hline
\end{tabular}

\section{(d) Emotional Network}

Average LAA > High LAA

\begin{tabular}{lllllll}
\hline High LAA > Average LAA & & & & & \\
\hline (1) Amygdala & $\mathrm{R}$ & 58 & 16 & -8 & -14 & $p<0.01$ \\
\hline (2) Mamillary body & $\mathrm{R}$ & 13 & 2 & -10 & -16 & $p<0.01$ \\
\hline
\end{tabular}

Table 3. Brain areas exhibiting increased functional connectivity for Average or High LAA group within the Independent Components distinguished by the group Tensor-ICA analysis; $\mathrm{x}, \mathrm{y}$ and $\mathrm{z}$ coordinates in MNI space, regions labelled according to Harvard-Oxford Cortical and Subcortical Structural Atlases and Juelich Histological Atlas (all implemented within FSLVIEW, part of FSL). 
(a)

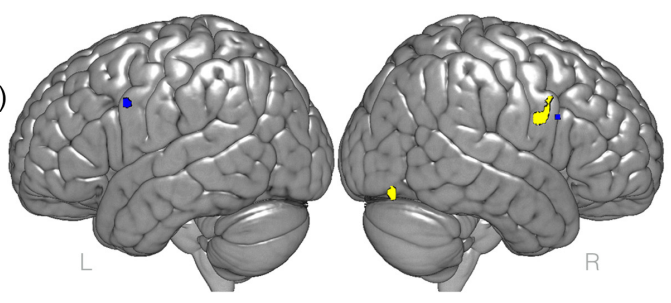

(c)

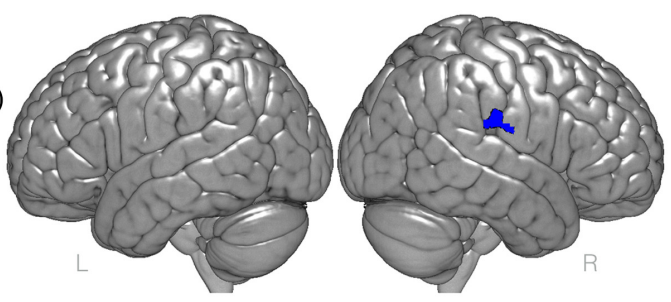

(b)
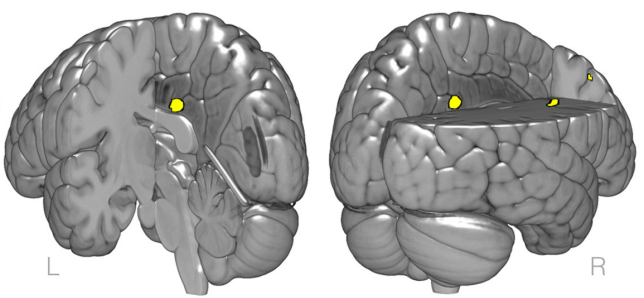

(d)

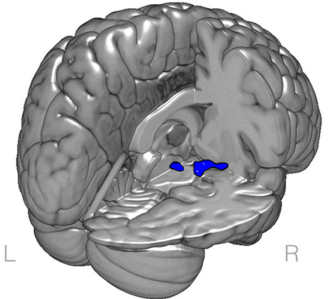

Average LAA > High LAA

High LAA > Average LAA

Figure 3. Results of the between-group analysis in the voxel-wise spatial distribution of the functional connectivity maps showing differences between High and Average LAA participants within the (a) task-positive/language network, (b) default mode network, (c) working memory network, and (d) emotional network. Results of two-sample unpaired t-tests are displayed at $p<.01$, TFCE-corrected. In blue, the results of the contrast testing for stronger connectivity for the High as compared to Average LAA participants are shown; in yellow, for the Average as compared to High LAA participants. Brain activations are displayed using MRIcroGL (http://www.mccauslandcenter.sc.edu/mricrogl/).

\section{Discussion and conclusions}

In an effort to advance the understanding of brain function and organisation coupled with second language learning, the present study set out to describe brain connectivity patterns in a group of adults learning novel grammar. Furthermore, we aimed at identifying neural mechanisms underlying individual variability in language acquisition by investigating network characteristics responsible for high abilities in grammar learning (operationalized by means of pre-testing participants' language analytical abilities). We collected functional connectivity data simultaneous to the learners acquiring new grammar rules and applied a data-driven analysis, looking for whole-brain connectivity patterns during the process of learning. It was expected that several distinct networks would be found: pertaining to the type of received input as well as representing intrinsic organisation of the brain found in resting-state and task related investigations alike (see e.g. $[24,25,28,49,92]$ ). We further investigated differences between two groups of participants recruited on the basis of their language analytical abilities and expected that the highly skilled learners would be characterised by stronger functional connectivity patterns, especially of syntax-related regions. Bilateral contribution from Broca's area and its righthemisphere homologue were predicted for the moderately skilled learners.

By extracting and identifying six independent components in the collected fMRI data, we showed multiple processes being present during acquisition of novel grammar, thus providing insight into the intricate and multi-layered nature of learning new language rules. Indeed, we found networks reproducing earlier resting-state investigations, such as the task-positive and the default mode network, as well as correlated activations, which we assume were driven by the type of input received by the participants. Those included a working memory network, a set of co-activated visual areas, a cerebellar and an emotional network. 
In the following sections, we will discuss the possible significance of each of the identified networks for the grammar learning process, and the established markers of inter-individual learners' differences.

\subsection{Task-positive/language network}

The first of the extracted ICs, the task-positive or language network, comprised of regions related to language processing and could - typical for language-related activations - be distinguished by asymmetry from the left hemisphere. The component's map was consistent with the task-positive network (cf. [24,49]) and showed overlap with the set of regions described by Dodel et al. [21] as syntaxrelated, but also extended it. We noted more frontal activations, in the frontal pole and middle frontal gyrus, with the addition of activity in language areas in the parietal and temporal lobes and in the occipital lobe and cerebellum.

One of our hypotheses, formulated on the basis of Antonenko et al.'s [17] findings (see sections 1.1 and 1.4 above), assumed bilateral contributions from Broca's area and its right-hemisphere homologue in case of the moderately skilled learners. The observed group differences in this component indicated increased involvement in the network from areas extending from the traditionally defined Broca's area to the more posterior structures of the ventrolateral prefrontal cortex. In terms of lateralisation of these prefrontal contributions to the network, the observed results were opposite to the findings of Antonenko et al.'s [17]: bilateral contributions were observed only among participants with high analytical abilities. The Average LAA group in our study demonstrated stronger functional connectivity within this network from the right precentral gyrus bordering on pars opercularis in the IFG and the right occipital fusiform gyrus (see Figure 3a). One possible explanation for this discrepancy in lateralisation effects might be the fact that Antonenko et al. [17] investigated the ability to acquire grammatical rules among older adults, who previously have been shown to process information differently from young people: bilateral activations of the prefrontal cortex have been found on a number of tasks where young adults display unilateral activations [93]. Antonenko et al. [17] suggested that the explanation for the increased functional correlation between bilateral prefrontal areas in their study, lay in the a lack of inhibition between those functionally connected brain regions. Our data suggest, that among young adults the engagement of areas surrounding Broca's region and its right-hemisphere homologue to the network do not reflect a lack of predisposition to acquire novel grammar (which could be expected on the basis of Antonenko's study). Rather, the learning skills of the High LAA group were reflected in stronger contributions of both the left and the right frontal regions.

In terms of the exact localisation of the clusters in the prefrontal cortex, in the context of syntax processing, the areas immediately posterior to IFG have been previously established to be activated during phonological processing [32], to reflect increased perceptual demands [94], or to underlie general structural/sequential processing of input [95]. Indeed, they have also been shown to be involved in artificial grammar learning experiments [96]. They seem then to have a supporting role for the core syntactic areas localised in BA 44 [97]. Friederici [98] suggested further that in comparison with BA 44/45, the ventral premotor cortex regions are phylogenetically older, hence they are more likely to be involved in syntactic processing on a lower level (i.e. based on local probabilities vs. hierarchical structures). Since our participants were in an initial phase of processing the presented input syntactically, it seems understandable 
that the regions exhibiting increased connectivity within the task-positive network were not yet localised within the core area underlying syntactic processing. Note that the highly skilled participant, in comparison with the average, exhibited increased contributions from areas closer to the traditionally defined Broca's region. It might thus be the case, that one's analytical abilities determine the involvement of the prefrontal cortex during language learning: the closer to the core, the easier it might be to learn the new syntactic rules. On the other hand, what seems to underlie lower analytical abilities, is the engagement of areas typical to lower level processing (word recognition), as was observed among the Average LAA participants.

Another possible explanation for such differences in network engagement between the High and the Average LAA participants might be their utilising different strategies for the learning task. Previous research has pointed to rulebased and memory-based learning as two possible mechanisms underlying acquisition of novel grammar rules [37,99-101], with memory-based strategies being most prevalent in initial stages of learning [101]. In particular, the finding of stronger functional connectivity of the right occipital fusiform gyrus to the task positive network of the Average LAA group might suggest that one's analytical abilities play a role in determining the strategies employed and thus brain structures involved in novel grammar learning.

\subsection{Default mode network}

The default mode network was first discovered as a set of cortical areas increasing their activation levels during rest as opposed to performance of cognitive tasks [102], cf. [76], thus reflecting a default state of neuronal activity of the human brain [51,73,76,103,104]. Further investigations suggested the DMN to underpin internally-directed processes, such as internal attention [49], integration of cognitive and emotional stimuli [74], mind wandering [105] and representation of the world around us [103]. A network reproducing the set of regions traditionally described as belonging to the DMN was also observed in our data, with the contribution of the subcallosal cortex. Since the subcallosal cortex has been described as a part of motivation/drive circuit of the brain [106], its contribution to the DMN derived from data acquired during a learning task, is not entirely surprising (see also the discussion on the emotional network in section 4.6 below). One possible explanation for this finding is that our acquisition paradigm (data were collected simultaneously to the task, and not during rest) might cause a shift in the balance between the task-positive and default mode network (cf. [107]), resulting in additional areas contributing to the DMN. However, considering the fact that the subcallosal cortex has previously been reported to contribute to the DMN uniquely in clinically depressed individuals [108], its role in task-related functional connectivity merits further investigation.

When it comes to differences in the DMN between the High and Average LAA participants, we observed stronger contributions to the network for the Average as compared to the High LAA group. The difference was localised in three regions: the posterior cingulate gyrus, the paracingulate gyrus bordering on anterior cingulate and in a cluster in the right frontal pole and middle frontal gyrus (see Figure 3b). This finding of increased connectivity within the DMN only among the Average LAA participants, and in regions marking on-going execution of cognitive processes (cf. e.g. [82,109]), might point to the conclusion that intrinsic characteristics of one's DMN determine the predispositions to efficiently 
acquire novel languages. During the language learning task at hand, the Average LAA participants engaged more neural resources for internally-directed processes, such as mind wandering, instead of utilising them for the resolution of cognitive operations demanded by the task.

\subsection{Working memory network}

The set of areas identified as the next component was consistent with the working memory network as described by Owen et al. [82] with the addition of activations in the superior parietal lobule, the supramarginal gyrus and the thalamus. All those areas show increased activity in cognitively demanding tasks, in particular verbal ones [82-84]. Working memory capacity, defined as the ability to build, maintain and update information [110], is undeniably critical for a learning task in which rules have to be continuously discovered and revised (see e.g. [111-113] for additional support for the links between working memory and rule learning). Interestingly, besides the working memory activations we noted activity in the left temporo-occipital fusiform cortex, the localization of which corresponds to the visual word form area (cf. $[85,86]$ ). This collaboration between the fronto-parietal working memory system and the VWFA can be traced back to the type of input employed in the task: building, maintaining and updating the grammar rules driven by the fronto-parietal working memory system most likely proceeds on the basis of the recognition of letters and words driven by the VWFA.

Within the working memory IC, the central opercular/secondary somatosensory cortex in the right hemisphere was found to exhibit increased functional connectivity for the High as compared to the Average LAA group (see Figure 3a). The fact that the engagement of a network consistent with working memory related processes, differed as a function of pre-tested analytical abilities, does not come as a surprise. Language analytical abilities can be seen as domain-specific proxy for fluid intelligence (since the latter is defined as variation in general reasoning and the ability to solve novel problems, cf. [114]) and the links between fluid intelligence and working memory are well established in the literature (e.g. [114-117]). Increased connectivity within this IC in the right central opercular/secondary somatosensory cortex can be seen as advantageous when it comes to determining one's abilities to acquire new languages, in particular since the role of the secondary somatosensory cortex has been described to consist of perception, integration and categorisation of incoming input [118].

\subsection{Visual areas}

A network responsible for lower-level processes present during grammar learning comprised of areas specialised for visual processing. Since the stimulus material in our study was presented visually, the presence of a network including activations in the lateral occipital cortex and occipital pole seems clear to interpret. Moreover, the network reproduces findings from resting-state studies employing analytical approaches similar to ours (e.g. [24,25,28]), where visual areas were consistently forming separate components identified by the analyses. Contrary to some investigations though, the ICA in our study produced one visual component map, whereas Beckmann et al. [24], Damoiseaux et al. [25] and Smith et al. [28], report two visual maps separating more lateral and more medial visual areas. The explanation for this difference may lie in our analysis employing a smaller number of pre-set components: ICA decompositions with 
higher numbers of components produce higher separation of the functional subnetworks [28].

\subsection{Cerebellar network}

Cerebellar involvement in language functions is one of coordination and automatisation. For example, by means of the "cerebellar deficit framework", Nicolson et al. [119] explain dyslexia: such a deficit might cause problems with automatisation of learned skills (such as articulation and spelling) and result in impairments of reading and writing (see [89] for a review). Moreover, the cerebellum has important function for - among others - working memory during processing [120] and meta-linguistic and higher-level language abilities [121,122], cf. De Smet et al. [88].

In our data, the cerebellar activations were coupled with a set of regions (right IFG and the basal ganglia structures, pallidum and putamen) previously put forward to form the procedural learning system [87]. Additionally, we observed activity in the thalamus, which is the node connecting the cerebellum to the cortex (cf. [88]), and in the right temporal pole. Note that the structural connectivity of the temporal pole, as shown by tractography, suggests its role as a convergence centre, with important implications in language and multimodal semantic processing [123]. The contribution of the left temporal fusiform gyrus (involved in the recognition of written words, cf. [124,125] can be related to the type of received input during the task (strings of written words). Taken together, the connectivity between the temporal pole and temporal fusiform gyrus might point to the process of assignment of semantic features to letter strings, which are then taken up by the procedural learning system in order to be processed as a grammar in a more holistic way.

No group differences were found in a comparison between High and Average LAA participants in this network. In other words, when it comes to coordination and automatisation processes, both groups exhibited brain activity of similar profiles, making the cerebellar involvement not being driven by the participants' language analytical abilities.

\subsection{Emotional network}

The last of the identified components comprised of areas corresponding to the regions together forming "the emotional brain" circuitry, dedicated to processing and regulating emotions [90,91]. This finding is in accordance with the view that emotion and cognition, by means of strong interaction and integration, together contribute to behaviour [90]. Furthermore, the activations found within this network extended to such structures as the inferior olivary nucleus and the red nucleus, which (through their connectivity with the cerebellum via the climbing fibres system) play an important role in procedural learning (cf. [126]).

In the context of language learning, the emotional circuits seem in particular crucial for the control of motivation and (intrinsic) reward mechanisms (cf. [127]). The presence of an emotional network in our data could mean that acquisition of new grammatical rules in part relies on subcortical, emotional circuits. The group differences in this network were found for the High as compared to Average LAA participants. The learners with higher language analytical abilities showed stronger contributions within this network from the right amygdala and mammillary body in the right hemisphere (see Figure 3d). The amygdalar involvement during the task can be explained by its role in encoding and retrieving of emotionally significant information (e.g. [129]). Furthermore, the 
amygdala seems to enable memory consolidation of emotionally salient information through its interactions with the hippocampus [54,130], which also contributed to the network in question. Mammillary bodies, and their projections to the anterior thalamus, are important for episodic memory [131]. Taken together, these increased contributions to the emotional network from the High LAA participants suggest that highly skilled learners might have associated more emotional valence with the material being learned. It might also be the case that the subcortical emotional brain circuitry of individuals with higher language abilities exhibits a more integrated organisation in the presence of a language learning task.

\subsection{The interconnected brain during novel language rule learning}

By investigating functional connectivity during a novel grammar-learning task by means of a data-driven, unbiased whole-brain approach, we found a set of networks bearing significant importance for such a process of learning. The collected data were decomposed into maps, each representing a separate putative cognitive process present during the task. We found both internally oriented (default mode network) activations next to externally, task-oriented ones. The information with which the participants were confronted had to be continuously manipulated and updated, by means of working memory. At the same time the learning process was coordinated by the cerebellum; all this in the presence of emotional activity, driving the will to learn.

Apart from decomposing the learning process into several cognitive subprocesses, we investigated differences between participants with varying degrees of language analytical abilities. The obtained results did not corroborate our first hypothesis, predicting bilateral contribution from Broca's area and its righthemisphere homologue for the moderately skilled learners. Instead, we found contributions from areas adjacent to Broca's right-hemisphere homologue for both groups, stronger involvement from the right occipital fusiform gyrus in case of Average LAA participants and stronger contribution of the areas adjacent to left Broca's area in case of the High.

We further hypothesised that the highly skilled learners would be distinguished by stronger functional connectivity patterns, especially of syntax-related regions. Examination of the results of the between-group analyses lets us conclude that this hypothesis is confirmed: the majority of the results pointed to stronger connectivity within networks for High LAA participants. Average LAA participants exhibited stronger functional connectivity in the default mode network, which makes us believe that since DMN is normally deactivated during a task, the stronger connectivity of this network during a task is not advantageous for the demands of the task at hand. In sum, we demonstrated that in terms of functional connectivity, the engagement of brain's networks during grammar acquisition is coupled with one's language learning abilities.

Although informative as to the nature of distinct processes being present during acquisition of language-like rules, there are still open questions deriving from this research. For example, the matter of interactions and collaborations between different networks present during learning awaits further investigation. Although extracted as Independent Components, the networks are indeed connected to each other and by means of other methodological approaches, the interactions between them can be brought to light (cf. e.g. [28]). Furthermore, it merits to be explored how other building blocks of language learning (such as acquisition of new vocabulary items (cf. e.g.[132]) and inter-individual differences 
in rote learning memory) are represented at the neural level, employing the same or similar methodologies. Also, research should try to elucidate whether the connectivity strength within various networks, as found in our study, bears consequence for structural connectivity measures and other indexes of anatomical variability.

\section{Acknowledgements}

This research was supported by the Leiden University Centre for Linguistics and the NWO Graduate Programme. The funding sources had no involvement in the preparation of the manuscript, or in the decision to submit the article for publication. We thank Bahar Azizi, Ferdi van de Kamp and Bobby Ruijgrok for their help in data acquisition, and Bertram Opitz for advice on creation of the stimulus material. We are grateful to Egbert Lakke for advice on neuroanatomy, and to two anonymous reviewers for their insightful feedback on an earlier version of the manuscript.

\section{References}

[1] S.L. Bressler, V. Menon, Large-scale brain networks in cognition: emerging methods and principles, Trends Cogn. Sci. 14 (2010) 277-290.

[2] S.L. Bressler, Large-scale cortical networks and cognition, Brain Res. Rev. 20 (1995) 288-304.

[3] A.D. Friederici, W. Singer, Grounding language processing on basic neurophysiological principles, Trends Cogn. Sci. 19 (2015) 1-10. doi:10.1016/j.tics.2015.03.012.

[4] M.M. Mesulam, From sensation to cognition, Brain. 121 (1998) 1013-1052. doi:10.1093/brain/121.6.1013.

[5] W. Singer, Synchronization of cortical activity and its putative role in information processing and learning., Annu. Rev. Physiol. 55 (1993) 349374. doi:10.1146/annurev.physiol.55.1.349.

[6] S. Weiss, H.M. Mueller, The contribution of EEG coherence to the investigation of language, Brain Lang. 85 (2003) 325-343. doi:10.1016/S0093-934X(03)00067-1.

[7] L. García-Pentón, A. Pérez Fernández, Y. Iturria-Medina, M. GillonDowens, M. Carreiras, Anatomical connectivity changes in the bilingual brain., Neuroimage. $84 \quad$ (2014) 495-504. doi:10.1016/j.neuroimage.2013.08.064.

[8] D. López-Barroso, M. Catani, P. Ripollés, F. Dell'Acqua, A. RodríguezFornells, R. de Diego-Balaguer, Word learning is mediated by the left arcuate fasciculus., Proc. Natl. Acad. Sci. U. S. A. 110 (2013) 13168-73. doi:10.1073/pnas.1301696110.

[9] P. Loui, H.C. Li, G. Schlaug, White matter integrity in right hemisphere predicts pitch-related grammar learning., Neuroimage. 55 (2011) 500-507. doi:10.1016/j.neuroimage.2010.12.022.

[10] M. Catani, M.P.G. Allin, M. Husain, L. Pugliese, M.M. Mesulam, R.M. Murray, D.K. Jones, Symmetries in human brain language pathways 
correlate with verbal recall., Proc. Natl. Acad. Sci. U. S. A. 104 (2007) 17163-8. doi:10.1073/pnas.0702116104.

[11] S.G. Mohades, E. Struys, P. Van Schuerbeek, K. Mondt, P. Van De Craen, R. Luypaert, DTI reveals structural differences in white matter tracts between bilingual and monolingual children., Brain Res. 1435 (2012) 7280. doi:10.1016/j.brainres.2011.12.005.

[12] M. Stein, A. Federspiel, T. Koenig, M. Wirth, W. Strik, R. Wiest, D. Brandeis, T. Dierks, Structural plasticity in the language system related to increased second language proficiency., Cortex. 48 (2012) 458-65. doi:10.1016/j.cortex.2010.10.007.

[13] H. Xiang, D. Dediu, L. Roberts, D.G. Norris, P. Hagoort, Language Aptitude , Working Memory, and IQ in the Perisylvian Language Network, Lang. Learn. 62 (2012) 110-130.

[14] A. Pérez, M. Gillon Dowens, N. Molinaro, Y. Iturria-Medina, P. Barraza, L. García-Pentón, M. Carreiras, Complex brain network properties in late L2 learners and native speakers., Neuropsychologia. 68 (2015) 209-217.

[15] S. Reiterer, E. Pereda, J. Bhattacharya, On a Possible Relationship between Linguistic Expertise and EEG Gamma Band Phase Synchrony., Front. Psychol. 2 (2011) 334. doi:10.3389/fpsyg.2011.00334.

[16] S. Reiterer, E. Pereda, J. Bhattacharya, Measuring second language proficiency with EEG synchronization: how functional cortical networks and hemispheric involvement differ as a function of proficiency level in second language speakers, Second Lang. Res. 25 (2009) 77-106. doi:10.1177/0267658308098997.

[17] D. Antonenko, M. Meinzer, R. Lindenberg, A.V. Witte, A. Flöel, Grammar learning in older adults is linked to white matter microstructure and functional connectivity., Neuroimage. $62 \quad$ (2012) 1667-74. doi:10.1016/j.neuroimage.2012.05.074.

[18] L. Ghazi Saidi, V. Perlbarg, G. Marrelec, M. Pélégrini-Issac, H. Benali, A.I. Ansaldo, Functional connectivity changes in second language vocabulary learning., Brain Lang. 124 (2013) 56-65. doi:10.1016/j.bandl.2012.11.008.

[19] N. Ventura-Campos, A. Sanjuán, J. González, M.-Á. Palomar-García, A. Rodríguez-Pujadas, N. Sebastián-Gallés, G. Deco, C. Ávila, Spontaneous brain activity predicts learning ability of foreign sounds., J. Neurosci. 33 (2013) 9295-305. doi:10.1523/JNEUROSCI.4655-12.2013.

[20] D. Cordes, V.M. Haughton, K. Arfanakis, G.J. Wendt, P.A. Turski, C.H. Moritz, M.A. Quigley, M.E. Meyerand, Mapping Functionally Related Regions of Brain with Functional Connectivity MR Imaging, (2000) 16361644.

[21] S. Dodel, N. Golestani, C. Pallier, V. Elkouby, D. Le Bihan, J.-B. Poline, Condition-dependent functional connectivity: syntax networks in bilinguals., Philos. Trans. R. Soc. Lond. B. Biol. Sci. 360 (2005) 921-935.

[22] K. Veroude, D.G. Norris, E. Shumskaya, M. Gullberg, P. Indefrey, Functional connectivity between brain regions involved in learning words of a new language., Brain Lang. $113 \quad$ (2010) 21-7. doi:10.1016/j.bandl.2009.12.005. 
[23] J. Yang, K.M. Gates, P. Molenaar, P. Li, Neural changes underlying successful second language word learning: An fMRI study, J. Neurolinguistics. 33 (2015) 29-49. doi:10.1016/j.jneuroling.2014.09.004.

[24] C.F. Beckmann, M. DeLuca, J.T. Devlin, S.M. Smith, Investigations into resting-state connectivity using independent component analysis., Philos. Trans. R. Soc. Lond. B. Biol. Sci. 360 (2005) 1001-1013.

[25] J.S. Damoiseaux, S.A.R.B. Rombouts, F. Barkhof, P. Scheltens, C.J. Stam, S.M. Smith, C.F. Beckmann, Consistent resting-state networks across healthy subjects, Proc. Natl. Acad. Sci. U. S. A. 103 (2006) 13848-13853. doi:10.1073/pnas.0601417103.

[26] E. Van Diessen, S.J.H. Diederen, K.P.J. Braun, F.E. Jansen, C.J. Stam, Functional and structural brain networks in epilepsy: What have we learned?, Epilepsia. 54 (2013) 1855-1865.

[27] J. Rissman, A. Gazzaley, M. D'Esposito, Measuring functional connectivity during distinct stages of a cognitive task., Neuroimage. 23 (2004) 752-63. doi:10.1016/j.neuroimage.2004.06.035.

[28] S.M. Smith, P.T. Fox, K.L. Miller, D.C. Glahn, P.M. Fox, C.E. Mackay, N. Filippini, K.E. Watkins, R. Toro, A.R. Laird, C.F. Beckmann, Correspondence of the brain's functional architecture during activation and rest., Proc. Natl. Acad. Sci. U. S. A. 106 (2009) 13040-5. doi:10.1073/pnas.0905267106.

[29] B. Biswal, F.Z. Yetkin, V.M. Haughton, J.S. Hyde, Functional connectivity in the motor cortex of resting human brain using echo-planar MRI., Magn. Reson. Med. 34 (1995) 537-41.

[30] K.M. Petersson, V. Folia, P. Hagoort, What artificial grammar learning reveals about the neurobiology of syntax, Brain Lang. 120 (2012) 83-95. doi:10.1016/j.bandl.2010.08.003.

[31] A.S. Reber, Implicit learning of artificial grammars, J. Verbal Learning Verbal Behav. 6 (1967) 855-863.

[32] K.M. Petersson, P. Hagoort, The neurobiology of syntax: beyond string sets, Philos. Trans. R. Soc. B Biol. Sci. 367 (2012) 1971-1983.

[33] A.D. Friederici, K. Steinhauer, E. Pfeifer, Brain signatures of artificial language processing: evidence challenging the critical period hypothesis, Proc. Natl. Acad. Sci. U. S. A. 99 (2002) 529-534.

[34] B. Opitz, A.D. Friederici, Neural basis of processing sequential and hierarchical syntactic structures., Hum. Brain Mapp. 28 (2007) 585-92. doi:10.1002/hbm.20287.

[35] B. Opitz, A.D. Friederici, Interactions of the hippocampal system and the prefrontal cortex in learning language-like rules, Neuroimage. 19 (2003) 1730-1737. doi:10.1016/S1053-8119(03)00170-8.

[36] B. Opitz, N.K. Ferdinand, A. Mecklinger, Timing matters: the impact of immediate and delayed feedback on artificial language learning., Front. Hum. Neurosci. 5 (2011) 8. doi:10.3389/fnhum.2011.00008.

[37] B. Opitz, A.D. Friederici, Brain correlates of language learning: the neuronal dissociation of rule-based versus similarity-based learning., J. Neurosci. 24 (2004) 8436-40. doi:10.1523/JNEUROSCI.2220-04.2004. 
[38] M.F.A. a Hauser, J. Hofmann, B. Opitz, Rule and similarity in grammar: Their interplay and individual differences in the brain, Neuroimage. 60 (2012) 2019-2026. doi:10.1016/j.neuroimage.2012.02.016.

[39] G. Brod, B. Opitz, Does it really matter? Separating the effects of musical training on syntax acquisition., Front. Psychol. 3 (2012) 543. doi:10.3389/fpsyg.2012.00543.

[40] Z. Dörnyei, P. Skehan, Individual differences in second language learning., in: C.J. Doughty, M.H. Long (Eds.), Handb. Second Lang. Acquis., Blackwell Publishing, Oxford, 2003: pp. 549-630.

[41] R. Ellis, The Study of Second Language Acquisition, 2nd Ed., Oxford University Press, Oxford, 2008.

[42] M. Sawyer, L. Ranta, Aptitude, individual differences, and instructional design, in: P. Robinson (Ed.), Cogn. Second Lang. Instr., Cambridge University Press, 2001: pp. 319-353.

[43] P.M. Meara, Llama Language Aptitude Tests, Lognostics, Swansea, 2005.

[44] P. Skehan, Theorizing and updating aptitude, in: P. Robinson (Ed.), Individ. Differ. Instr. Lang. Learn., John Benjamins Publishing Company, Philadelphia/Amsterdam, 2002: pp. 69-93.

[45] B. Harley, D. Hart, Language Aptitude And Second Language Proficiency In Classroom Learners Of Different Starting Ages, Stud. Second Lang. Acquis. 19 (1997).

[46] R. Erlam, Language aptitude and its relationship to instructional effectiveness in second language acquisition, Lang. Teach. Res. 9 (2005) 147-172.

[47] Y. Yilmaz, Relative Effects of Explicit and Implicit Feedback: The Role of Working Memory Capacity and Language Analytic Ability, Appl. Linguist. 34 (2012) 344-368. doi:10.1093/applin/ams044.

[48] C.F. Beckmann, M. Jenkinson, M.W. Woolrich, T.E.J. Behrens, D.E. Flitney, J.T. Devlin, S.M. Smith, Applying FSL to the FIAC data: modelbased and model-free analysis of voice and sentence repetition priming., Hum. Brain Mapp. 27 (2006) 380-91. doi:10.1002/hbm.20246.

[49] M.D. Fox, A.Z. Snyder, J.L. Vincent, M. Corbetta, D.C. Van Essen, M.E. Raichle, The human brain is intrinsically organized into dynamic, anticorrelated functional networks., Proc. Natl. Acad. Sci. U. S. A. 102 (2005) 9673-8. doi:10.1073/pnas.0504136102.

[50] M.-J. Van Tol, I.M. Veer, N.J. Van der Wee, A. Aleman, M. Van Buchem, S.R.B. Rombouts, F.G. Zitman, D.J. Veltman, T. Johnstone, Whole-brain functional connectivity during emotional word classification in medicationfree Major Depressive Disorder: Abnormal salience circuitry and relations to positive emotionality., NeuroImage. Clin. 2 (2013) 790-6. doi:10.1016/j.nicl.2013.05.012.

[51] M.P. Van Den Heuvel, H.E. Hulshoff Pol, Exploring the brain network: A review on resting-state fMRI functional connectivity, Eur. Neuropsychopharmacol. $20 \quad$ (2010) 519-534. doi:10.1016/j.euroneuro.2010.03.008.

[52] M. De Luca, C.F. Beckmann, N. De Stefano, P.M. Matthews, S.M. Smith, 
fMRI resting state networks define distinct modes of long-distance interactions in the human brain., Neuroimage. 29 (2006) 1359-67. doi:10.1016/j.neuroimage.2005.08.035.

[53] C.F. Beckmann, S.M. Smith, Tensorial extensions of independent component analysis for multisubject FMRI analysis., Neuroimage. 25 (2005) 294-311. doi:10.1016/j.neuroimage.2004.10.043.

[54] I.M. Veer, The stress connection: neuroimaging studies of emotion circuits in social stress, personality, and stress-related psychopathology, Leiden Institute for Brain and Cognition (LIBC), Faculty of Social and Behavioural Sciences, Leiden University, 2015.

[55] O. Kepinska, M. de Rover, J. Caspers, N.O. Schiller, On neural correlates of individual differences in novel grammar learning: an fMRI study, Neuropsychologia. (2016). doi:10.1016/j.neuropsychologia.2016.06.014.

[56] N.A. Macmillan, C.D. Creelman, Detection Theory: A User's Guide, Psychology Press, 2005.

[57] IBM SPSS, IBM SPSS, IBM Softw. Bus. Anal. (2012).

[58] M. Jenkinson, C.F. Beckmann, T.E.J. Behrens, M.W. Woolrich, S.M. Smith, FSL, Neuroimage. 62 (2012) 782-790.

[59] M. Jenkinson, P. Bannister, M. Brady, S.M. Smith, Improved optimisation for the robust and accurate linear registration and motion correction of brain images., Neuroimage. 17 (2002) 825-841.

[60] S.M. Smith, Fast robust automated brain extraction, Hum. Brain Mapp. 17 (2002) 143-155.

[61] M. Jenkinson, S. Smith, A global optimisation method for robust affine registration of brain images, Med. Image Anal. 5 (2001) 143-156.

[62] A. Hyvärinen, Fast and robust fixed-point algorithms for independent component analysis., IEEE Trans. Neural Netw. 10 (1999) 626-34. doi:10.1109/72.761722.

[63] C.F. Beckmann, S.M. Smith, Probabilistic independent component analysis for functional magnetic resonance imaging., IEEE Trans. Med. Imaging. 23 (2004) 137-52. doi:10.1109/TMI.2003.822821.

[64] C.F. Beckmann, C.E. Mackay, N. Filippini, S.M. Smith, Group comparison of resting-state FMRI data using multi-subject ICA and dual regression, Neuroimage. 47 (2009) S39-S41. doi:10.1016/S1053-8119(09)71511-3.

[65] N. Filippini, B.J. MacIntosh, M.G. Hough, G.M. Goodwin, G.B. Frisoni, S.M. Smith, P.M. Matthews, C.F. Beckmann, C.E. Mackay, Distinct patterns of brain activity in young carriers of the APOE-epsilon4 allele., Proc. Natl. Acad. Sci. U. S. A. 106 (2009) 7209-14. doi:10.1073/pnas.0811879106.

[66] S.M. Smith, T.E. Nichols, Threshold-free cluster enhancement: addressing problems of smoothing, threshold dependence and localisation in cluster $\begin{array}{lllll}\text { inference., } & \text { Neuroimage. } & 44 & \text { (2009) } & \text { 83-98. }\end{array}$ doi:10.1016/j.neuroimage.2008.03.061.

[67] C.A. Seger, C.M. Cincotta, The roles of the caudate nucleus in human classification learning., J. Neurosci. $25 \quad$ (2005) 2941-51. 
doi:10.1523/JNEUROSCI.3401-04.2005.

[68] J.A. Grahn, J.A. Parkinson, A.M. Owen, The cognitive functions of the caudate nucleus., Prog. Neurobiol. $86 \quad$ (2008) 141-55. doi:10.1016/j.pneurobio.2008.09.004.

[69] J. Crinion, R. Turner, A. Grogan, T. Hanakawa, U. Noppeney, J.T. Devlin, T. Aso, S. Urayama, H. Fukuyama, K. Stockton, K. Usui, D.W. Green, C.J. Price, Language control in the bilingual brain., Science (80-. ). 312 (2006) 1537-40. doi:10.1126/science.1127761.

[70] A. Hervais-Adelman, B. Moser-Mercer, C.M. Michel, N. Golestani, fMRI of Simultaneous Interpretation Reveals the Neural Basis of Extreme Language Control., Cereb. Cortex. 25 (2014) 4727-39. doi:10.1093/cercor/bhu158.

[71] J. Abutalebi, D.W. Green, Control mechanisms in bilingual language production: Neural evidence from language switching studies, Lang. Cogn. Process. 23 (2008) 557-582. doi:10.1080/01690960801920602.

[72] A. Oh, E.G. Duerden, E.W. Pang, The role of the insula in speech and language processing., Brain Lang. 135 (2014) 96-103. doi:10.1016/j.bandl.2014.06.003.

[73] M.E. Raichle, A.M. MacLeod, A.Z. Snyder, W.J. Powers, D.A. Gusnard, G.L. Shulman, A default mode of brain function., Proc. Natl. Acad. Sci. U. S. A. 98 (2001) 676-82. doi:10.1073/pnas.98.2.676.

[74] M.D. Greicius, B. Krasnow, A.L. Reiss, V. Menon, Functional connectivity in the resting brain: a network analysis of the default mode hypothesis., Proc. Natl. Acad. Sci. U. S. A. 100 (2003) 253-258. doi:10.1073/pnas.0135058100.

[75] M.D. Greicius, G. Srivastava, A.L. Reiss, V. Menon, Default-mode network activity distinguishes Alzheimer's disease from healthy aging: evidence from functional MRI., Proc. Natl. Acad. Sci. U. S. A. 101 (2004) 4637-42. doi:10.1073/pnas.0308627101.

[76] M.E. Raichle, The Brain's Default Mode Network., Annu. Rev. Neurosci. 38 (2015) 433-47. doi:10.1146/annurev-neuro-071013-014030.

[77] L. Wang, F. Zou, Y. Shao, E. Ye, X. Jin, S. Tan, D. Hu, Z. Yang, Disruptive changes of cerebellar functional connectivity with the default mode network in schizophrenia, Schizophr. Res. 160 (2014) 67-72. doi:10.1016/j.schres.2014.09.034.

[78] C. Habas, N. Kamdar, D. Nguyen, K. Prater, C.F. Beckmann, V. Menon, M.D. Greicius, Distinct cerebellar contributions to intrinsic connectivity networks., J. Neurosci. 29 (2009) 8586-94. doi:10.1523/JNEUROSCI.186809.2009.

[79] F.M. Krienen, R.L. Buckner, Segregated fronto-cerebellar circuits revealed by intrinsic functional connectivity., Cereb. Cortex. 19 (2009) 2485-97. doi:10.1093/cercor/bhp135.

[80] R.L. Buckner, F.M. Krienen, A. Castellanos, J.C. Diaz, B.T.T. Yeo, The organization of the human cerebellum estimated by intrinsic functional connectivity., J. Neurophysiol. $106 \quad$ (2011) 2322-45. doi:10.1152/jn.00339.2011. 
[81] J.X. O'Reilly, C.F. Beckmann, V. Tomassini, N. Ramnani, H. JohansenBerg, Distinct and overlapping functional zones in the cerebellum defined by resting state functional connectivity., Cereb. Cortex. 20 (2010) 953-65. doi:10.1093/cercor/bhp157.

[82] A.M. Owen, K.M. McMillan, A.R. Laird, E. Bullmore, N-back working memory paradigm: a meta-analysis of normative functional neuroimaging studies., Hum. Brain Mapp. 25 (2005) 46-59. doi:10.1002/hbm.20131.

[83] M. Koenigs, A.K. Barbey, B.R. Postle, J. Grafman, Superior Parietal Cortex Is Critical for the Manipulation of Information in Working Memory, J. Neurosci. 29 (2009) 14980-14986. doi:10.1523/JNEUROSCI.370609.2009.

[84] I. Deschamps, S.R. Baum, V.L. Gracco, On the role of the supramarginal gyrus in phonological processing and verbal working memory: evidence from rTMS studies., Neuropsychologia. $53 \quad$ (2014) 39-46. doi:10.1016/j.neuropsychologia.2013.10.015.

[85] S. Dehaene, L. Cohen, The unique role of the visual word form area in reading., Trends Cogn. Sci. $15 \quad$ (2011) 254-62. doi:10.1016/j.tics.2011.04.003.

[86] B.D. McCandliss, L. Cohen, S. Dehaene, The visual word form area: expertise for reading in the fusiform gyrus, Trends Cogn. Sci. 7 (2003) 293299. doi:10.1016/S1364-6613(03)00134-7.

[87] M.T. Ullman, Contributions of memory circuits to language: the declarative / procedural model, Cognition. 92 (2004) 231-270. doi:10.1016/j.cognition.2003.10.008.

[88] H.J. De Smet, P. Paquier, J. Verhoeven, P. Mariën, The cerebellum: its role in language and related cognitive and affective functions., Brain Lang. 127 (2013) 334-42. doi:10.1016/j.bandl.2012.11.001.

[89] R.I. Nicolson, A.J. Fawcett, Dyslexia, dysgraphia, procedural learning and the cerebellum, Cortex. $47 \quad$ (2011) 117-127. doi:10.1016/j.cortex.2009.08.016.

[90] L. Pessoa, On the relationship between emotion and cognition., Nat. Rev. Neurosci. 9 (2008) 148-58. doi:10.1038/nrn2317.

[91] I.M. Veer, N.Y.L. Oei, P. Spinhoven, M.A. van Buchem, B.M. Elzinga, S.A.R.B. Rombouts, Endogenous cortisol is associated with functional connectivity between the amygdala and medial prefrontal cortex., $\begin{array}{llll}\text { Psychoneuroendocrinology. } & 37 & \text { (2012) }\end{array}$ doi:10.1016/j.psyneuen.2011.12.001.

[92] M.D. Fox, M.E. Raichle, Spontaneous fluctuations in brain activity observed with functional magnetic resonance imaging, Nat Rev Neurosci. 8 (2007) 700-711.

[93] P.M. Greenwood, R. Parasuraman, Neuronal and cognitive plasticity: a neurocognitive framework for ameliorating cognitive aging., Front. Aging Neurosci. 2 (2010) 150. doi:10.3389/fnagi.2010.00150.

[94] A.D. Friederici, The brain basis of language processing: from structure to function., Physiol. $\quad$ Rev. $91 \quad$ (2011) 1357-92. doi:10.1152/physrev.00006.2011. 
[95] M. Hoen, M. Pachot-Clouard, C. Segebarth, P.F. Dominey, When Broca experiences the Janus syndrome: an ER-fMRI study comparing sentence comprehension and cognitive sequence processing., Cortex. 42 (2006) 60523.

[96] J. Bahlmann, R.I. Schubotz, A.D. Friederici, Hierarchical artificial grammar processing engages Broca's area, Neuroimage. 42 (2008) 525534. doi:10.1016/j.neuroimage.2008.04.249.

[97] T. Goucha, A.D. Friederici, The language skeleton after dissecting meaning: A functional segregation within Broca's Area., Neuroimage. 114 (2015) 294-302. doi:10.1016/j.neuroimage.2015.04.011.

[98] A.D. Friederici, Broca's Area and the Ventral Premotor Cortex in Language: Functional Differentiation and Specificity, Cortex. 42 (2006) 472-475. doi:10.1016/S0010-9452(08)70380-0.

[99] T.J. Domangue, R.C. Mathews, R. Sun, L.G. Roussel, C.E. Guidry, Effects of model-based and memory-based processing on speed and accuracy of grammar string generation., J. Exp. Psychol. Learn. Mem. Cogn. 30 (2004) 1002-11. doi:10.1037/0278-7393.30.5.1002.

[100] R.C. Mathews, R. Buss, W.B. Stanley, F. Blanchard-Fields, J.R. Cho, B. Druhan, Role of Implicit and Explicit Processes in Learning From Examples, J. Exp. Psychol. Learn. Mem. Cogn. 15 (1989) 1083-1100.

[101] B. Opitz, J. Hofmann, Concurrence of rule- and similarity-based mechanisms in artificial grammar learning., Cogn. Psychol. 77 (2015) 7799. doi:10.1016/j.cogpsych.2015.02.003.

[102] G.L. Shulman, J.A. Fiez, M. Corbetta, R.L. Buckner, F.M. Miezin, M.E. Raichle, S.E. Petersen, Common Blood Flow Changes across Visual Tasks: II. Decreases in Cerebral Cortex., J. Cogn. Neurosci. 9 (1997) 648-63. doi:10.1162/jocn.1997.9.5.648.

[103] D.A. Gusnard, M.E. Raichle, Searching for a baseline: Functional imaging and the resting human brain, Nat Rev Neurosci. 2 (2001) 685-694. doi:10.1038/35094500.

[104] M.E. Raichle, A.Z. Snyder, A default mode of brain function: a brief history of an evolving idea., Neuroimage. 37 (2007) 1083-90; discussion 1097-9. doi:10.1016/j.neuroimage.2007.02.041.

[105] M.F. Mason, M.I. Norton, J.D. Van Horn, D.M. Wegner, S.T. Grafton, C.N. Macrae, Wandering Minds: The Default Network and StimulusIndependent Thought, Science (80-. ). $315 \quad$ (2007) 393-395. doi:10.1126/science.1131295.

[106] N.D. Volkow, G.-J. Wang, J.S. Fowler, D. Tomasi, F. Telang, R. Baler, Addiction: decreased reward sensitivity and increased expectation sensitivity conspire to overwhelm the brain's control circuit., Bioessays. 32 (2010) 748-55. doi:10.1002/bies.201000042.

[107] I. Marchetti, E.H.W. Koster, E.J. Sonuga-Barke, R. De Raedt, The Default Mode Network and recurrent depression: A neurobiological model of cognitive risk factors, Neuropsychol. Rev. 22 (2012) 229-251. doi:10.1007/s11065-012-9199-9.

[108] M.D. Greicius, B.H. Flores, V. Menon, G.H. Glover, H.B. Solvason, H. 
Kenna, A.L. Reiss, A.F. Schatzberg, Resting-state functional connectivity in major depression: abnormally increased contributions from subgenual cingulate cortex and thalamus., Biol. Psychiatry. 62 (2007) 429-37. doi:10.1016/j.biopsych.2006.09.020.

[109] A. Fornito, M. Yücel, S. Wood, G.W. Stuart, J.-A. Buchanan, T. Proffitt, V. Anderson, D. Velakoulis, C. Pantelis, Individual differences in anterior cingulate/paracingulate morphology are related to executive functions in healthy males., Cereb. Cortex. 14 (2004) 424-31.

[110] O. Wilhelm, A. Hildebrandt, K. Oberauer, What is working memory capacity, and how can we measure it?, Front. Psychol. 4 (2013) 433. doi:10.3389/fpsyg.2013.00433.

[111] K.I. Martin, N.C. Ellis, The Roles Of Phonological Short-Term Memory And Working Memory In L2 Grammar And Vocabulary Learning, Stud. Second Lang. Acquis. 34 (2012) 379-413. doi:10.1017/S0272263112000125.

[112] R. De Diego-Balaguer, D. Lopez-Barroso, Cognitive and Neural Mechanisms Sustaining Rule Learning From Speech, Lang. Learn. 60 (2010) 151-187. doi:10.1111/j.1467-9922.2010.00605.x.

[113] N.C. Ellis, Working Memory in the Acquisition of Vocabulary and Syntax: Putting Language in Good Order, Q. J. Exp. Psychol. Sect. A. 49 (1996) 234-250. doi:10.1080/713755604.

[114] G.C. Burgess, J.R. Gray, A.R.A. Conway, T.S. Braver, Neural mechanisms of interference control underlie the relationship between fluid intelligence and working memory span., J. Exp. Psychol. Gen. 140 (2011) 674-692. doi:10.1037/a0024695.

[115] P.L. Ackerman, M.E. Beier, M.O. Boyle, Working memory and intelligence: the same or different constructs?, Psychol. Bull. 131 (2005) 30-60. doi:10.1037/0033-2909.131.1.30.

[116] J. Duncan, M. Schramm, R. Thompson, I. Dumontheil, Task rules, working memory, and fluid intelligence., Psychon. Bull. Rev. 19 (2012) 864-870.

[117] J.T. Shelton, E.M. Elliott, R.A. Matthews, B.D. Hill, W.D. Gouvier, The relationships of working memory, secondary memory, and general fluid intelligence: working memory is special., J. Exp. Psychol. Learn. Mem. Cogn. 36 (2010) 813-820.

[118] D. Crystal, R. Varley, Introduction to Language Pathology, John Wiley \& Sons, 2013.

[119] R.I. Nicolson, A.J. Fawcett, P. Dean, Time estimation deficits in developmental dyslexia: evidence of cerebellar involvement., Proc. Biol. Sci. 259 (1995) 43-7. doi:10.1098/rspb.1995.0007.

[120] P. Marien, S. Engelborghs, F. Fabbro, P.P. De Deyn, The lateralized linguistic cerebellum: a review and a new hypothesis., Brain Lang. 79 (2001) 580-600. doi:10.1006/brln.2001.2569.

[121] M. Cook, B. Murdoch, L. Cahill, B. Whelan, Higher-level language deficits resulting from left primary cerebellar lesions, Aphasiology. 18 (2004) 771784. doi:10.1080/02687030444000291.

[122] B.-M. Whelan, B. Murdoch, Unravelling subcortical linguistic substrates: Comparison of thalamic versus cerebellar cognitive-linguistic regulation 
mechanisms, $\quad$ Aphasiology. $\quad 19 \quad$ (2005) 1097-1106. doi:10.1080/02687030500174050.

[123] R.J. Binney, G.J.M. Parker, M.A. Lambon Ralph, Convergent connectivity and graded specialization in the rostral human temporal lobe as revealed by diffusion-weighted imaging probabilistic tractography, J. Cogn. Neurosci. 24 (2012) 1998-2014. doi:10.1162/jocn.

[124] K.S. Weiner, K. Zilles, The anatomical and functional specialization of the fusiform gyrus., Neuropsychologia. doi:10.1016/j.neuropsychologia.2015.06.033.

[125] B.A. Kirchhoff, A.D. Wagner, A. Maril, C.E. Stern, Prefrontal-Temporal Circuitry for Episodic Encoding and Subsequent Memory, J. Neurosci. 20 (2000) 6173-6180.

[126] A. Siegel, H.N. Sapru, Essential Neuroscience, Lippincott Williams \& Wilkins, 2011.

[127] P. Ripollés, J. Marco-Pallarés, U. Hielscher, A. Mestres-Missé, C. Tempelmann, H.-J. Heinze, A. Rodríguez-Fornells, T. Noesselt, The Role of Reward in Word Learning and Its Implications for Language Acquisition, Curr. Biol. 24 (2014) 2606-2611. doi:10.1016/j.cub.2014.09.044.

[128] S. Syal, B.L. Finlay, Thinking outside the cortex: social motivation in the evolution and development of language., Dev. Sci. 14 (2011) 417-30.

[129] M. Sarter, H.J. Markowitsch, Involvement of the amygdala in learning and memory: a critical review, with emphasis on anatomical relations., Behav. Neurosci. 99 (1985) 342-80.

[130] J.L. McGaugh, The amygdala modulates the consolidation of memories of emotionally arousing experiences., Annu. Rev. Neurosci. 27 (2004) 1-28. doi:10.1146/annurev.neuro.27.070203.144157.

[131] S.D. Vann, Re-evaluating the role of the mammillary bodies in memory, Neuropsychologia. $48 \quad$ (2010) 2316-2327. doi:10.1016/j.neuropsychologia.2009.10.019.

[132] D. López-Barroso, P. Ripollés, J. Marco-Pallarés, B. Mohammadi, T.F. Münte, A.-C. Bachoud-Lévi, A. Rodriguez-Fornells, R. de Diego-Balaguer, Multiple brain networks underpinning word learning from fluent speech revealed by independent component analysis, Neuroimage. 110 (2015) 182-193. doi:10.1016/j.neuroimage.2014.12.085. 